PAPER

\title{
Insulin-like growth factor I promoter polymorphism, risk of stroke, and survival after stroke: the Rotterdam study
}

\author{
M J E van Rijn, A J C Slooter, M J Bos, C F B S Catarino, P J Koudstaal, A Hofman, M M B Breteler, \\ C M van Duijn
}

J Neurol Neurosurg Psychiatry 2006;77:24-27. doi: 10.1136/jnnp.2005.067447

See end of article for authors' affiliations

.....................

Correspondence to: Professor C M van Duijn, Department of Epidemiology and Biostatistics, Erasmus Medical Center, PO Box $1738,3000 \mathrm{DR}$ Rotterdam, The Netherlands; c.vanduijn@ erasmusmc.nl

Received 10 March 2005 Revised version received 22 June 2005

Accepted 23 June 2005

\begin{abstract}
Background and purpose: Low levels of insulin-like growth factor I (IGF-I) predispose to atherosclerosis and may therefore increase the risk of stroke. Low levels have also been found to influence the outcome of cardiovascular and cerebrovascular disease. A polymorphism in the promoter region of the IGF-I gene influences IGF-I levels. Non-carriers of the 192 bp allele have lower levels of IGF-I compared with 192 bp allele carriers. We studied the IGF-I polymorphism in relation to the risk of stroke and survival after stroke. Methods: We studied 6808 subjects of the Rotterdam Study, who were followed for the occurrence of stroke and death after stroke. Subjects were grouped according to the 192 bp allele of IGF-I into noncarriers, heterozygotes, and homozygotes. The risk of stroke and survival after stroke was studied using Cox regression analysis, adjusting for age and sex, with homozygotes for the wildtype allele as the reference.

Results: Non-carriers had a relative risk of $0.8(95 \% \mathrm{Cl}: 0.6$ to 1.0$)$ for the occurrence of any stroke and 0.7 (95\% Cl: 0.5 to 1.0) for ischaemic stroke. For non-carriers, the relative risk of death after any stroke was 1.5 (95\% Cl: 1.0 to 2.2). After an ischaemic stroke, this relative risk was $1.5(95 \% \mathrm{Cl}: 0.9$ to 2.6 ) and after a haemorrhagic stroke $5.2(95 \% \mathrm{Cl}: 1.3$ to 21.5$)$.

Conclusions: Our study suggests that IGF-I is a significant determinant of survival after stroke.
\end{abstract}

in sulin-like growth factor I (IGF-I) appears to be involved in the development of atherosclerosis and cardiovascular disease because of its role in tissue repair and cell proliferation. ${ }^{1-4}$ Also, IGF-I induces the synthesis of elastin and prevents apoptosis of vascular smooth muscle cells. ${ }^{5-7}$ Therefore, low levels might be a risk factor for stroke. Expression of IGF-I is increased after hypoxic injury in regions with neuronal $\operatorname{loss}^{8}$ and animal studies have found that administration of IGF-I reduces infarct volume and improves neurological function after ischaemia. ${ }^{8-10}$ This suggests that IGF-I levels may be involved in outcome after stroke. Indeed, it was found that IGF-I levels are lower after cerebral ischaemia, ${ }^{11}{ }^{12}$ and low levels are associated with a poor outcome after ischaemic stroke ${ }^{11}$ as well as myocardial infarction (MI). ${ }^{13}$ We have previously found that a polymorphism in the promoter region of the IGF-I gene, located on chromosome 12q, is associated with plasma IGF-I levels. Non-carriers of the $192 \mathrm{bp}$ (wildtype) allele of this polymorphism have lower plasma IGF-I levels compared with $192 \mathrm{bp}$ allele carriers. ${ }^{14}$ Also, non-carriers of the $192 \mathrm{bp}$ allele were found to have an increased risk of $\mathrm{MI}^{14}$ and an increase in mean intima-media thickness of the common carotid arteries and mean pulse wave velocity. ${ }^{15}$ We studied the association between the IGF-I promoter polymorphism and the risk of stroke, as well as survival after stroke, for any stroke, ischaemic stroke, and haemorrhagic stroke.

\section{METHODS}

\section{Study population}

The present study was performed as part of the Rotterdam Study, an ongoing prospective population based cohort study on chronic and disabling diseases in the elderly. ${ }^{16}$ The medical ethics committee of Erasmus Medical Center, Rotterdam, approved the study. Participants gave written informed consent and permission to retrieve information from treating physicians. A total of 7983 subjects participated in this study
(78\% response rate), all aged 55 years and over at baseline. IGF-I genotyping was successfully performed in 7012 (68.5\%) individuals using frozen serum samples collected at baseline. The genotyping success rate was $87.8 \%$. No DNA was available for 948 subjects and genotyping failed in 23 subjects. Serum IGF-I levels were assessed in a randomly selected subgroup of 406 subjects, as described earlier. ${ }^{17}$ Participants with a stroke before baseline were excluded from the analyses $(n=258)$. Our study population therefore comprised 6808 individuals (85\%). The sample appeared to be random, as we did not find differences between participants with and without a known IGF-I genotype with regard to demographic or cardiovascular characteristics.

\section{Measurements and diagnosis of stroke}

Baseline data were collected from 1990 until 1993. Measurements and definitions of body mass index (BMI), hypertension, cholesterol levels, and diabetes mellitus are described elsewhere. ${ }^{18}$ A previous stroke was determined during the baseline interview by asking "did you ever suffer from a stroke, diagnosed by a physician?". Medical records of subjects who answered "yes" were checked in order to verify the diagnosis. ${ }^{19}$

Follow up started at baseline and lasted until 1 January 2002 for the present study. Once subjects enter the Rotterdam Study, they are continuously monitored for major events through automated linkage of the study database with files from general practitioners (GPs) and the municipality. Also nursing home physicians' files are scrutinised. For reported events, additional information (including brain images) is obtained from hospital records. Trained research physicians reviewed information on all possible strokes and transient ischaemic attacks. An experienced stroke

Abbreviations: $\mathrm{BMI}$, body mass index; $\mathrm{CV}$ death, cardiovascular death after stroke; IGF-I, insulin-like growth factor I; GP, general practitioner; $\mathrm{MI}$, myocardial infarction 
Table 1 Baseline characteristics of the study population stratified by IGF-1 genotype

\begin{tabular}{|c|c|c|c|}
\hline & \multicolumn{3}{|l|}{ IGF-1 } \\
\hline & $192 / 192$ & $192 /-$ & $-1-$ \\
\hline Number of subjects & 2985 & 3014 & 809 \\
\hline Age & $69.5 \pm 9.2$ & $69.3 \pm 9.2$ & $69.5 \pm 9.4$ \\
\hline Sex, $\mathrm{n}(\%$ male $)$ & 1191 (39.9) & $1202(39.9)$ & $310(38.3)$ \\
\hline $\mathrm{SBP}(\mathrm{mm} \mathrm{Hg})$ & $139.1 \pm 22.1$ & $139.3 \pm 22.5$ & $139.0 \pm 22.3$ \\
\hline $\mathrm{DBP}(\mathrm{mm} \mathrm{Hg})$ & $73.6 \pm 11.4$ & $73.9 \pm 11.8$ & $73.3 \pm 11.4$ \\
\hline Hypertension n (\%) & $981(33.8)$ & 998 (34.2) & $256(32.7)$ \\
\hline Diabetes, $\mathrm{n}(\%)$ & $287(9.6)$ & $316(10.5)$ & $74(9.1)$ \\
\hline Current smoking, & $656(22.7)$ & $676(23.3)$ & $159(20.2)$ \\
\hline $\begin{array}{l}\text { BMI }\left(\mathrm{kg} / \mathrm{m}^{2}\right) \\
\text { Total cholesterol }\end{array}$ & $\begin{array}{l}26.3 \pm 3.8 \\
6.6 \pm 1.3\end{array}$ & $\begin{array}{l}26.2 \pm 3.6 \\
6.6 \pm 1.2\end{array}$ & $\begin{array}{l}26.2 \pm 3.7 \\
6.6 \pm 1.2\end{array}$ \\
\hline$(\mathrm{mmol} / \mathrm{l})$ & & & \\
\hline $\begin{array}{l}\text { HDL cholesterol } \\
\text { (mmol/l) }\end{array}$ & $1.3 \pm 0.4$ & $1.3 \pm 0.4$ & $1.4 \pm 0.4$ \\
\hline $\begin{array}{l}\text { Serum total IGF-I } \\
\text { levels }(\mathrm{nmol} / \mathrm{l})^{*}\end{array}$ & $\begin{array}{l}18.5 \pm 7.0 \\
(n=130)\end{array}$ & $\begin{array}{l}18.4 \pm 7.8 \\
(n=136)\end{array}$ & $\begin{array}{l}14.6 \pm 5.8 \\
(n=140) \dagger\end{array}$ \\
\hline
\end{tabular}

*Determined in a randomly drawn subgroup; †significantly different from homozygous and heterozygous carriers $(p<0.001)$.

All values are presented as unadjusted means \pm standard deviation or total numbers (percentages). DBP, diastolic blood pressure; SBP, systolic blood pressure.

neurologist (PJK) verified all diagnoses. Subarachnoid haemorrhages and retinal strokes were excluded. Follow up was completed until January 1, 2001 for $97.1 \%$ of all potential person years. ${ }^{20}$ Ischaemic strokes were diagnosed when a patient had typical symptoms and a CT or MRI performed within 4 weeks ruled out other diagnoses or when indirect evidence (deficit limited to one limb or completely resolved within $72 \mathrm{~h}$, atrial fibrillation in the absence of anticoagulants) suggested an ischaemic stroke. Haemorrhagic stroke was diagnosed when a relevant haemorrhage was shown on CT or MRI scan, or when the subject permanently lost consciousness or died within hours after onset of focal signs. If a stroke did not match these criteria, it was classified as unspecified. Neuroimaging was available for $61 \%$ of all events. For ischaemic stroke, this was $91 \%$ and for haemorrhagic stroke $78 \%$. Ischaemic stroke was subclassified into large vessel disease, small vessel disease, and stroke due to a cardio-embolic source using the TOAST criteria. ${ }^{21}$

Information on mortality was obtained from the GPs and hospitals and also regularly from the municipal health authorities in Rotterdam. Two research physicians independently coded all events according to the International Classification of Diseases, 10th edition (ICD-10).22 We defined cardiovascular mortality as death from ischaemic heart disease (I20-25), ventricular fibrillation (I49), congestive heart failure (I50), cerebrovascular disease (I60-67), peripheral artery disease (I70-74), hypertensive heart disease (I10-15), disorders of the aorta, arteries, arterioles, and

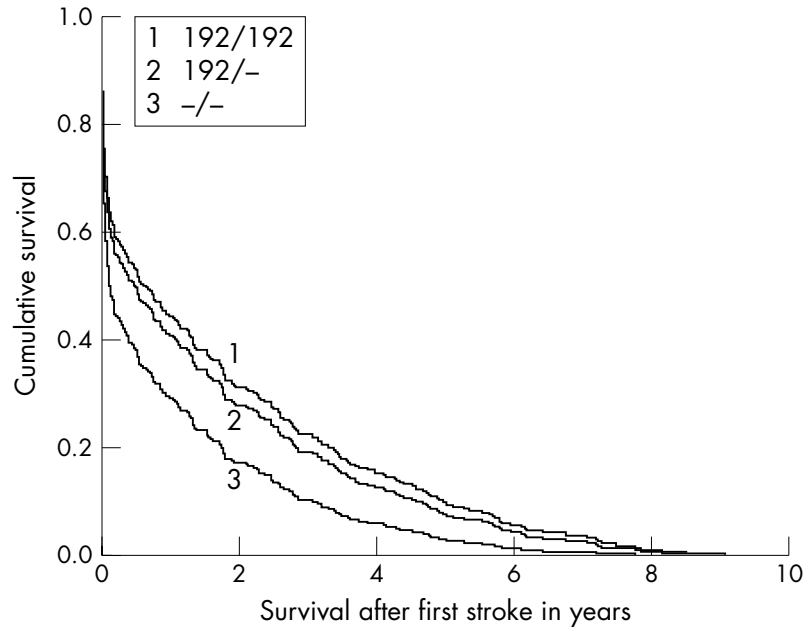

Figure 1 Cumulative survival after any stroke.

capillaries (I70-79) or mesenteric atherosclerosis (K55), sudden cardiac death (I46), or sudden death unknown (R96). The IGF-I gene promoter polymorphism was genotyped as described previously, without knowledge of the clinical diagnosis. ${ }^{14}$

\section{Data analysis}

Hardy-Weinberg equilibrium of the IGF-I promotor polymorphism genotypes was tested using the GENEPOP package. ${ }^{23}$ Baseline characteristics were compared using univariate ANOVA or $\chi^{2}$ statistics. Cox proportional hazards regression analysis was used to assess the relative risk of stroke, as well as the relative risk of death after stroke, with the homozygous carriers of the wildtype allele (192 bp) as the reference group (SPSS 11.0; SPSS, Chicago, IL). We tested the proportional hazard assumption using the log-log survival curve (SPSS 11.0) and goodness-of-fit using S-PLUS 6.0 (MathSoft, Cambridge, MA). All analyses were adjusted for age and sex.

\section{RESULTS}

All IGF-I genotype and allele proportions were in HardyWeinberg equilibrium. Table 1 shows the baseline characteristics of the study population stratified by IGF-I genotype. Serum total IGF-I levels were significantly lower in noncarriers of the $192 \mathrm{bp}$ allele compared with homozygous and heterozygous carriers $(\mathrm{p}<0.001)$. No significant differences were observed between the genotype groups and age, sex, or other cardiovascular risk factors.

During a mean follow up of 8 years, we observed 637 incident stroke cases in our study population. We classified 357 as ischaemic stroke, 59 as haemorrhagic stroke, and 221

Table 2 Relative risk of stroke and stroke subtypes in relation to IGF-1 genotype

\begin{tabular}{|c|c|c|c|c|c|c|}
\hline & \multicolumn{6}{|c|}{ IGF-I genotype } \\
\hline & \multicolumn{2}{|c|}{$192 / 192(n=2985)$} & \multicolumn{2}{|c|}{$192 /-(n=3014)$} & \multicolumn{2}{|c|}{$-/-(n=809)$} \\
\hline & $\mathrm{n}$ & RR & $\mathrm{n}$ & RR $(95 \%$ Cl) & $\mathrm{n}$ & RR $(95 \% \mathrm{Cl})$ \\
\hline All strokes & 266 & 1.0 (reference) & 229 & $0.9(0.7$ to 1.0$)$ & 56 & $0.8(0.6$ to 1.0$)$ \\
\hline Ischaemic strokes & 156 & 1.0 (reference) & 131 & $0.8(0.7$ to 1.1$)$ & 30 & $0.7(0.5$ to 1.0$)$ \\
\hline Haemorrhagic strokes & 22 & 1.0 (reference) & 25 & $1.1(0.6$ to 2.0$)$ & 6 & $1.0(0.4$ to 2.4$)$ \\
\hline Unspecified stroke & 88 & 1.0 (reference) & 73 & $0.8(0.6$ to 1.1$)$ & 20 & $0.8(0.5$ to 1.3$)$ \\
\hline Large vessel disease & 12 & 1.0 (reference) & 13 & $1.1(0.5$ to 2.4$)$ & 5 & $1.5(0.5$ to 4.4$)$ \\
\hline Cardio-embolic stroke & 26 & 1.0 (reference) & 22 & $0.8(0.5$ to 1.5$)$ & 8 & $1.1(0.5$ to 2.5$)$ \\
\hline Small vessel disease & 21 & 1.0 (reference) & 18 & $0.9(0.5$ to 1.6$)$ & 3 & $0.5(0.2$ to 1.7$)$ \\
\hline
\end{tabular}

$\mathrm{n}$, total number of cases; RR, relative risk with $95 \%$ confidence interval, adjusted for age and sex. 
Table 3 Relative risk of death after stroke in relation to IGF-1 genotype

\begin{tabular}{|c|c|c|c|c|c|c|c|c|}
\hline \multirow[b]{2}{*}{ IGF-1 } & \multicolumn{2}{|c|}{ Any stroke } & \multicolumn{2}{|c|}{ Ischaemic stroke } & \multicolumn{2}{|c|}{ Haemorrhagic stroke } & \multicolumn{2}{|c|}{ Unspecified } \\
\hline & $\mathbf{n}$ & $\operatorname{RR}(95 \% \mathrm{Cl})$ & $\mathbf{n}$ & $\operatorname{RR}(95 \% \mathrm{Cl})$ & $\mathbf{n}$ & $\operatorname{RR}(95 \% \mathrm{Cl})$ & $\mathbf{n}$ & $\operatorname{RR}(95 \% \mathrm{Cl})$ \\
\hline $192 / 192$ & 144 & 1.0 (reference) & 65 & 1.0 (reference) & 15 & 1.0 (reference) & 88 & 1.0 (reference) \\
\hline 192/- & 135 & $1.1(0.9$ to 1.4$)$ & 65 & $1.2(0.9$ to 1.8$)$ & 19 & $0.9(0.4$ to 1.9$)$ & 73 & $1.1(0.8$ to 1.7$)$ \\
\hline$-1-$ & 37 & $1.5(1.0 \text { to } 2.2)^{*}$ & 17 & $1.5(0.9$ to 2.6$)$ & 5 & $5.2(1.3$ to 21.5$) \dagger$ & 20 & $1.4(0.7$ to 2.4$)$ \\
\hline
\end{tabular}

$\mathrm{n}$, absolute numbers of death after stroke; RR, relative risk of death with $95 \%$ confidence intervals, adjusted for age and sex.

${ }^{*} \mathrm{p}<0.05$ compared with the reference group.

as unspecified. We had data on the IGF-I genotype for 551 $(86.5 \%)$ cases. This corresponds to the proportion of successfully performed genotyping in subjects overall (87.8\%). Table 2 shows the absolute numbers of strokes and risk estimates for each genotype group for any, ischaemic, haemorrhagic, and unspecified stroke. Non-carriers were found to have a risk of 0.8 (95\% CI: 0.6 to $1.0, p=0.05)$ for the occurrence of any stroke and 0.7 (95\% CI: 0.5 to 1.0, $\mathrm{p}=0.07$ ) for ischaemic stroke, compared with homozygote carriers of the $192 \mathrm{bp}$ allele. In this table, we also show the risk of subtypes of ischaemic stroke for different IGF-I genotypes. We found that non-carriers had a relative risk of large vessel disease of 1.5 (95\% CI: 0.5 to $4.4, p=0.4$ ), a relative risk of cardio-embolic stroke of 1.1 (95\% CI: 0.5 to $2.5, p=0.8)$, and a relative risk of small vessel disease of 0.5 (95\% CI: 0.2 to $1.7, p=0.3$ ). All values were adjusted for age and sex. Adjusting for additional risk factors (smoking status, diabetes mellitus, hypertension, BMI, and total cholesterol level) did not alter these results.

Figure 1 shows the cumulative survival in years after any stroke. The cumulative survival is lowest for non-carriers. Also, it is shown that the effect of the IGF-1 genotype on survival is strongest in the first months after a stroke. The same was found for survival after ischaemic stroke, haemorrhagic stroke, and unspecified stroke. Table 3 describes the absolute numbers of death after a stroke and the relative risk for each genotype. For non-carriers, we found that the relative risk of death after any stroke was 1.5 (95\% CI: 1.0 to $2.2, p=0.03$ ), after ischaemic stroke 1.5 (95\% CI: 0.9 to 2.6 , $\mathrm{p}=0.1)$, and after haemorrhagic stroke 5.2 (95\% CI: 1.3 to 21.5, $\mathrm{p}=0.02$ ) compared with homozygous carriers. Numbers were too small for analyses in subclassifications of ischaemic stroke. The risk estimates remained similar after adjusting for the additional risk factors.

We also analysed the effect of IGF-I on survival in the overall population, with exclusion of prevalent and incident stroke (not shown). The mean survival time in the cohort (until death or end of follow up) was 8.5 years for all three genotypes, adjusted for age and sex. We found a relative risk of 1.0 (95\% CI: 0.9 to 1.1) for heterozygote carriers and of 1.0 (95\% CI: 0.8 to 1.1 ) for non-carriers compared with homozygous carriers of the $192 \mathrm{bp}$ allele.

Next, we analysed the effect of the IGF-I genotype on cardiovascular death after stroke (CV death). For any stroke, ischaemic stroke, and haemorrhagic stroke, the absolute numbers of CV death were $20(35.7 \%), 8(26.7 \%)$, and 4 $(66.7 \%)$ in non-carriers, compared with 71 (26.7\%), 32
$(20.5 \%)$ and $8(36.4 \%)$ in homozygous carriers, respectively (table 4). The highest relative risk for CV death was 3.0 (95\% CI: 0.6 to 14.5), which was found in non-carriers after haemorrhagic stroke. However, this finding was not significant. Also, the relative risk of CV death after any, ischaemic, or unspecified stroke, was not significant (table 4). The proportional hazard assumption was met for all previously shown analyses.

\section{DISCUSSION}

This is the first investigation of the IGF-I promoter polymorphism and stroke. In this large population based cohort study, we found a protective effect for non-carriers of the $192 \mathrm{bp}$ allele of the IGF-I polymorphism on any and ischaemic stroke, although this was not significant. However, we found that it was a significant determinant of survival after stroke.

We cannot be sure that the ascertainment of stroke was complete. We retrieved information on stroke events through GPs, nursing home physicians, and participants. For clinical stroke, we believe we have complete ascertainment as we screened $97 \%$ of all GP and nursing home records for stroke events. The GP or nursing home physician would not have been informed only if a patient did not seek medical attention. Subclinical stroke however, presents without clinical symptoms. The only accurate way of verifying the completeness of stroke ascertainment would have been to perform an MRI or CT scan of the brain of every participant. Unfortunately, we did not have the equipment available to do this. However, as incompleteness of follow up of stroke will not have been dependent on genotype, we do not believe this has biased our results.

We previously observed that non-carriers of the $192 \mathrm{bp}$ allele had lower levels of IGF-I compared with heterozygous and homozygous carriers ${ }^{14}$ and that non-carriers of the IGF-I gene were at higher risk of developing atherosclerosis and MI. ${ }^{14}{ }^{15}$ In this study, for any and ischaemic stroke, we found a protective effect for non-carriers of the $192 \mathrm{bp}$ allele, although it was not significant. A possible explanation for this may be that non-carriers die from MI before they can develop a stroke. However, when we excluded prevalent and incident MI from the analyses, the relative risk for any and ischaemic stroke remained lower than 1. Another explanation may be that there are more causes of ischaemic stroke than large vessel atherosclerosis, which is in most cases the sole cause of MI. When we subtyped ischaemic stroke into

Table 4 Relative risk of cardiovascular death after stroke in relation to IGF-1 genotype

\begin{tabular}{|c|c|c|c|c|c|c|c|c|}
\hline \multirow[b]{2}{*}{ IGF-I } & \multicolumn{2}{|c|}{ Any stroke } & \multicolumn{2}{|c|}{ Ischaemic stroke } & \multicolumn{2}{|c|}{ Haemorrhagic stroke } & \multicolumn{2}{|c|}{ Unspecified } \\
\hline & $\mathbf{n}$ & $\operatorname{RR}(95 \% \mathrm{Cl})$ & $\mathbf{n}$ & $\operatorname{RR}(95 \% \mathrm{Cl})$ & $\mathbf{n}$ & $\operatorname{RR}(95 \% \mathrm{Cl})$ & $\mathbf{n}$ & $\operatorname{RR}(95 \% \mathrm{Cl})$ \\
\hline $192 / 192$ & 71 & 1.0 (reference) & 32 & 1.0 (reference) & 8 & 1.0 (reference) & 31 & 1.0 (reference) \\
\hline $192 /-$ & 64 & $0.9(0.7$ to 1.3$)$ & 23 & 0.7 (0.4 to 1.3$)$ & 12 & 0.7 (0.3 to 1.9$)$ & 29 & $1.45(0.9$ to 2.5$)$ \\
\hline$-/-$ & 20 & $1.3(0.8$ to 2.2$)$ & 8 & $1.3(0.6$ to 2.9$)$ & 4 & $3.0(0.6$ to 14.5$)$ & 8 & $1.2(0.5$ to 2.9$)$ \\
\hline
\end{tabular}

$\mathrm{n}$, absolute numbers of cardiovascular death after stroke; RR, relative risk of cardiovascular death with $95 \%$ confidence intervals, adjusted for age and sex. 
large vessel disease, small vessel disease, and cardio-embolic stroke according to the TOAST criteria, ${ }^{21}$ we found that noncarriers had a relative risk of large vessel disease of $1.5(95 \%$ CI: 0.5 to 4.4 ), a relative risk of cardio-embolic stroke of 1.1 (95\% CI: 0.5 to 2.5 ), and a relative risk of small vessel disease of 0.5 (95\% CI: 0.2 to 1.7). These results were not significant. However, they do show that non-carriers are at higher risk of stroke due to large vessel atherosclerosis (large vessel and cardio-embolic stroke), while this is not the case for small vessel stroke. This is also in concordance with earlier findings in this study population that non-carriers of the $192 \mathrm{bp}$ allele had a significantly higher common carotid intima-media thickness and a higher mean pulse wave velocity compared with homozygous carriers, ${ }^{15}$ suggesting an association between the IGF-I polymorphism and large vessel atherosclerosis.

Our study shows that the IGF-I promoter polymorphism is a significant determinant of survival after any and haemorrhagic stroke. As our analyses of haemorrhagic stroke were based on only five cases, the high estimate of the relative risk of 5.20 should be interpreted with caution. We could not find an association between IGF-I and survival in the overall population, which suggests that IGF-I is a determinant of survival specifically after stroke. The effect of IGF-I on survival after stroke may again be due to the association with MI, as it is one of the early causes of death after a stroke. ${ }^{24} 25$ In this study, we also found that the most important cause of death after a stroke was cardiovascular disease. After any stroke, $35.7 \%$ of the non-carriers died from cardiovascular disease compared with $26.7 \%$ of homozygous carriers. After haemorrhagic stroke, $67 \%$ of the non-carriers died of cardiovascular disease compared with $36.4 \%$ of homozygous carriers. Although the relative risk of $\mathrm{CV}$ death for noncarriers was greater than 1 for all stroke groups, these findings were not significant.

Another explanation could be related to the influence of the 192 bp allele on IGF-I secretion. IGF-I is involved in brain development. ${ }^{26}$ When neuronal loss occurs in the brain, levels of IGF-I increase. ${ }^{8}$ It has been shown that IGF-I reduces infarct volume and improves neurological function following cerebral ischaemia, as IGF-I protects neurons from apoptosis after diverse forms of injury. ${ }^{1027} 28$ Non-carriers have lower plasma IGF-I levels, ${ }^{14}$ and hence less neuroprotection, which may lead to more severe handicap and higher case fatality after ischaemic stroke. As we did not have data on handicap after stroke, we were not able to test this hypothesis.

Our findings are in line with a recent study by Denti et al who found that low levels of IGF-I are a significant predictor of poor outcome, mainly death, after ischaemic stroke. ${ }^{11}$ However, it was unclear if low IGF-I levels were a cause or a consequence of the poor outcome. Since we studied a gene associated with IGF-I levels, we believe we have strong evidence that low IGF-I levels are a cause and not a consequence of the disease outcome. This indicates that IGF-I might be used as a possible treatment for patients with stroke. Indeed, several animal studies have found that administration of IGF-I reduces infarct volume and improves neurological function after ischaemia. ${ }^{8-10} 27$

In conclusion, in this large prospective population based follow up study, we found that the IGF-I polymorphism was a significant determinant of survival after any stroke.

\footnotetext{
Authors' affiliations

M J E van Rijn, A J C Slooter, M J Bos, C F B S Catarino, A Hofman,

M M B Breteler, C M van Duijn, Department of Epidemiology and Biostatistics, Erasmus Medical Center, Rotterdam, The Netherlands P J Koudstaal, Department of Neurology, Erasmus Medical Center, Rotterdam, The Netherlands
}

This work was supported by the Netherlands Organization for Scientific Research (NWO) and the Municipality of Rotterdam

Competing interests: none declared

\section{REFERENCES}

1 Ferns GA, Motani AS, Anggard EE. The insulin-like growth factors: their putative role in atherogenesis. Artery $1991 ; 18(4): 197-225$

2 Janssen JA, Stolk RP, Pols HA, et al. Serum total IGF-I, free IGF-I, and IGFB-1 levels in an elderly population: relation to cardiovascular risk factors and disease. Arterioscler Thromb Vasc Biol 1998;18(2):277-82.

3 Bayes-Genis A, Conover CA, Schwartz RS. The insulin-like growth factor axis: a review of atherosclerosis and restenosis. Circ Res 2000;86(2):125-30.

4 Juul A, Scheike T, Davidsen M, et al. Low serum insulin-like growth factor I is associated with increased risk of ischaemic heart disease: a population-based case-control study. Circulation 2002;106(8):939-44.

5 Bai H, Pollman MJ, Inishi Y, et al. Regulation of vascular smooth muscle cell apoptosis. Modulation of bad by a phosphatidylinositol 3-kinase-dependent pathway. Circ Res 1999;85(3):229-37.

6 Foster J, Rich CB, Florini JR. Insulin-like growth factor I, somatomedin C, induces the synthesis of tropoelastin in aortic tissue. Coll Relat Res 1987;7(3): 161-9.

7 Conn KJ, Rich $C B$, Jensen $D E$, et al. Insulin-like growth factor-I regulates transcription of the elastin gene through a putative retinoblastoma control element. A role for Sp3 acting as a repressor of elastin gene transcription. J Biol Chem 1996;271(46):28853-60.

8 Gluckman P, Klempt N, Guan J, et al. A role for IGF-1 in the rescue of CNS neurons following hypoxic-ischaemic injury. Biochem Biophys Res Commun 1992; 182(2):593-9.

9 Liu XF, Fawcett JR, Thorne RG, et al. Non-invasive intranasal insulin-like growth factor-I reduces infarct volume and improves neurologic function in rats following middle cerebral artery occlusion. Neurosci Lett 2001;308(2):91-4.

10 Schabitz WR, Hoffmann TT, Heiland S, et al. Delayed neuroprotective effect of insulin-like growth factor-I after experimental transient focal cerebral ischemia monitored with MRI. Stroke 2001;32(5):1226-33.

11 Denti L, Annoni V, Cattadori E, et al. Insulin-like growth factor 1 as a predictor of ischaemic stroke outcome in the elderly. Am J Med 2004;1 17(5):312-7.

12 Schwab S, Spranger M, Krempien S, et al. Plasma insulin-like growth factor I and IGF binding protein 3 levels in patients with acute cerebral ischaemic injury. Stroke 1997;28(9): 1744-8.

13 Janssen JA, Lamberts SW. The role of IGF-I in the development of cardiovascular disease in type 2 diabetes mellitus: is prevention possible? Eur J Endocrinol 2002;146(4):467-77.

14 Vaessen N, Heutink P, Janssen JA, et al. A polymorphism in the gene for IGFI: functional properties and risk for type 2 diabetes and myocardial infarction. Diabetes 2001;50(3):637-42.

15 Schut AF, Janssen JA, Deinum J, et al. Polymorphism in the promoter region of the insulin-like growth factor I gene is related to carotid intima-media thickness and aortic pulse wave velocity in subjects with hypertension. Stroke 2003;34(7):1623-7.

16 Hofman A, Grobbee DE, de Jong PT, et al. Determinants of disease and disability in the elderly: the Rotterdam Elderly Study. Eur J Epidemiol 1991;7(4):403-22.

17 Rietveld I, Janssen JA, Hofman A, et al. A polymorphism in the IGF-I gene influences the age-related decline in circulating total IGF-I levels. Eur J Endocrinol 2003;148(2):171-5.

18 Hollander M, Bots ML, Del Sol Al, et al. Carotid plaques increase the risk of stroke and subtypes of cerebral infarction in asymptomatic elderly: the Rotterdam study. Circulation 2002;105(24):2872-7.

19 Bots ML, Looman SJ, Koudstaal PJ, et al. Prevalence of stroke in the general population. The Rotterdam Study. Stroke 1996;27(9):1499-501.

20 Clark TG, Altman DG, De Stavola BL. Quantification of the completeness of follow-up. Lancet 2002;359(9314):1309-10.

21 Adams HP Jr, Bendixen BH, Kappelle $\amalg$, et al. Classification of subtype of acute ischemic stroke. Definitions for use in a multicenter clinical trial. TOAST. Trial of Org 10172 in Acute Stroke Treatment. Stroke 1993;24(1):35-41.

22 WHO. International statistical classification of diseases and related health problems, 10th revision. WHO, Geneva, 1992.

23 Raymond M, Rousset F. GENEPOP-package, version 1.2. Montpelier, France: Laboratoire de Genetique et Environment, 1995.

24 Bronnum-Hansen $H$, Davidsen $M$, Thorvaldsen P. Long-term survival and causes of death after stroke. Stroke 2001;32(9):2131-6.

25 Hardie K, Hankey GJ, Jamrozik K, et al. Ten-year survival after first-ever stroke in the Perth community stroke study. Stroke 2003;34(8):1842-6.

26 Zackenfels K, Oppenheim RW, Rohrer H. Evidence for an important role of IGF-I and IGF-II for the early development of chick sympathetic neurons. Neuron 1995; 14(4):731-41.

27 Liu XF, Fawcett JR, Thorne RG, et al. Intranasal administration of insulin-like growth factor-I bypasses the blood-brain barrier and protects against focal cerebral ischaemic damage. J Neurol Sci $2001 ; 187(1-2): 91-7$.

28 Tagami M, Ikeda K, Nara Y, et al. Insulin-like growth factor-1 attenuates apoptosis in hippocampal neurons caused by cerebral ischemia and reperfusion in stroke-prone spontaneously hypertensive rats. Lab Invest 1997;76(5):613-7. 